\title{
OHMl: the ontology of host-microbiome interactions
}

\author{
Yongqun He ${ }^{1 *}$ DD, Haihe Wang ${ }^{1,2}$, Jie Zheng ${ }^{3}$, Daniel P. Beiting ${ }^{4}$, Anna Maria Masci ${ }^{5}$, Hong Yu ${ }^{1,6}$, Kaiyong Liu, \\ Jianmin Wu ${ }^{8}$, Jeffrey L. Curtis ${ }^{1,9}$, Barry Smith ${ }^{10}$, Alexander V. Alekseyenko ${ }^{11}$ and Jihad S. Obeid ${ }^{11}$
}

\begin{abstract}
Background: Host-microbiome interactions (HMls) are critical for the modulation of biological processes and are associated with several diseases. Extensive HMI studies have generated large amounts of data. We propose that the logical representation of the knowledge derived from these data and the standardized representation of experimental variables and processes can foster integration of data and reproducibility of experiments and thereby further HMl knowledge discovery.

Methods: Through a multi-institutional collaboration, a community-based Ontology of Host-Microbiome Interactions (OHMI) was developed following the Open Biological/Biomedical Ontologies (OBO) Foundry principles. As an OBO library ontology, OHMI leverages established ontologies to create logically structured representations of (1) microbiomes, microbial taxonomy, host species, host anatomical entities, and HMls under different conditions and (2) associated study protocols and types of data analysis and experimental results.
\end{abstract}

Results: Aligned with the Basic Formal Ontology, OHMl comprises over 1000 terms, including terms imported from more than 10 existing ontologies together with some $500 \mathrm{OHMl}$-specific terms. A specific OHMI design pattern was generated to represent typical host-microbiome interaction studies. As one major OHMl use case, drawing on data from over 50 peer-reviewed publications, we identified over 100 bacteria and fungi from the gut, oral cavity, skin, and airway that are associated with six rheumatic diseases including rheumatoid arthritis. Our ontological study identified new high-level microbiota taxonomical structures. Two microbiome-related competency questions were also designed and addressed. We were also able to use OHMI to represent statistically significant results identified from a large existing microbiome database data analysis.

Conclusion: OHMI represents entities and relations in the domain of HMls. It supports shared knowledge representation, data and metadata standardization and integration, and can be used in formulation of advanced queries for purposes of data analysis.

Keywords: Microbiome, Host-microbiome interaction, Ontology, Ontology of host-microbiome interactions, OHMI, Metadata, OBO Foundry, Rheumatic disease, Rheumatoid arthritis

\section{Background}

A microbiome is defined as a community of microbes (for example, bacteria) found in a particular habitat (for example, a human host) [1-3]. Microbiomes exist in and on human and other hosts, where they are crucial for active immunologic and physiological system development $[1,4-6]$. Research in host-microbiome interaction (HMI) has accelerated significantly in the past decade, as

\footnotetext{
* Correspondence: yongqunh@med.umich.edu

'University of Michigan Medical School, Ann Arbor, Ml 48109, USA Full list of author information is available at the end of the article
}

evidenced by the rise in the number of microbiomerelated publications indexed in PubMed (from 604 to over 11,500 in the ten years since 2018). This growing body of HMI studies and associated data pose significant challenges. For example, it can be difficult for investigators to achieve reproducible results across laboratories, and even more challenging to integrate data systematically across studies. To facilitate advanced data integration and knowledge discovery, several funding sources now require that data generated from funded research be structured to conform to the FAIR (Findable,

(C) The Author(s). 2019 Open Access This article is distributed under the terms of the Creative Commons Attribution 4.0 International License (http://creativecommons.org/licenses/by/4.0/), which permits unrestricted use, distribution, and 
Accessible, Interoperable, and Reusable) data principles [7]. To support data FAIRness and experimental reproducibility in HMI research, a strategy is needed to standardize the representation of the entities involved in HMI, including host and microbial organisms, microbial locations, and environments. As in other research areas, so also here: the lack of a comprehensive standardized representation of these entities prevents integration and systems-level analysis of the HMI data produced by different studies, laboratories and institutions.

An ontology is a human- and computer-interpretable representation of the types, properties, and interrelationships that exist in a particular domain [8]. Ontologies allow semantically-based reasoning by computer systems, and enable people and machines to make mutually supportive logical inferences. In biomedical research, ontologies have served for some 20 years as powerful tools for data classification, representation of standards, construction of knowledge bases, and enhanced search and analysis. Several microbiology-related ontologies exist, including the NCBI organismal classification (NCBITaxon) [9], the Uberon multi-species anatomy ontology (UBERON) [10] and the Environment Ontology (ENVO) [11]. These ontologies permit standardized representation of, respectively, host and microbial organisms, anatomic locations of microbes inside hosts, and microbiome environments. The Ontology for Microbial Phenotypes (OMP) standardizes phenotypic information relating to microbes [12]. The Ontology of Prokaryotic Phenotypic and Metabolic Characters (MicrO) covers the attributes of prokaryotes, the processes in which they participate, and the material entities (such as cell components, microbiological culture media and medium ingredients) with which they are associated in these processes [13]. Many of the terms in OMP and MicrO were themselves imported from existing OBO ontologies, including the Phenotypic Quality Ontology (PATO) [14], the Gene Ontology (GO) [15], Chemical Entities of Biological Interest (ChEBI) [16], the Protein Ontology (PR) [17], and the Ontology for Biomedical Investigations $(\mathrm{OBI})[18]$.

The above-mentioned ontologies provide components for the systematic representation of certain aspects of HMIs, but they do not cover, for example, HMIs - the interactions between hosts and microbiomes - themselves. They also do not cover the associations between HMIs and specific diseases (such as rheumatoid arthritis), or HMI investigation metadata. We have created the Ontology of Host-Microbiome Interactions (OHMI), therefore, not merely in order to incorporate terms in these specific areas, which are important foci of current microbiome research, but also to provide a single framework for systematic representation of all entities relevant to HMI.

\section{Methods}

\section{OHMI ontology development}

OHMI follows the Open Biological/Biomedical Ontologies (OBO) Foundry (http://www.obofoundry.org/) principles. For example, OHMI satisfies the openness and collaboration principles [19], in that it is based on an open discussion involving representatives from multiple disciplines engaged in microbiome research in which not only the scope of the ontology was identified but also the development strategy, design patterns, and initial use cases. The OHMI GitHub website (https://github.com/OHMI-ontology/OHMI) documents the successive versions of the ontology presented at the 23rd International Scientific Symposium on Biometrics (BioStat 2017), the Sixth Annual Workshop of the Clinical and Translational Science Ontology Group, and the Microbe 2018 meeting of the American Society of Microbiology (ASM).

OHMI uses the eXtensible Ontology development (XOD) methods [20], meaning that it reuses terms from existing ontologies and aligns all terms within a single semantic framework as defined by the Basic Formal Ontology (BFO) [21]. The Ontofox tool was used for extraction and reuse of terms from existing ontologies [22]. The Ontorat tool was used for generating new terms based on consensus ontology design patterns [23]. OHMI was formatted in the Web Ontology Language (OWL2), and the Protégé OWL Editor (version 5.0) [24] was used for manual editing. The HermiT reasoner (http://hermit-reasoner. $\mathrm{com} /$ ) tool was employed to detect inconsistencies or conflicts arising during development.

\section{Host-microbiome interaction minimal information collection and ontological representation}

All HMI-related data elements were first compiled in a spreadsheet from the literature, public resources, and use cases, then discussed by the community, and transformed into terms and relational expressions for inclusion in the ontology. Following the OBO Foundry principle of reuse, wherever a term was already defined in one or more existing ontologies (identified using Ontobee [25]) we imported the term into OHMI using what we deemed to be the most biologically accurate definition. Otherwise, we created a new term, which was either (1) included in OHMI or (2) suggested for inclusion in an appropriate higher-level OBO Foundry ontology in order to make it available for importing into OHMI.

\section{OHMI use case studies and evaluation}

Our major use case was the study of the association between microbiome profiles and rheumatic diseases. Rheumatic diseases include conditions causing chronic, often intermittent pain affecting the joints and/or connective tissues such as rheumatoid arthritis (RA), 
ankylosing spondylitis (AS), and systemic lupus erythematosus (SLE). In this study, we manually curated published rheumatic disease-related HMI data from peer-reviewed publications.

We have also defined and used two competency questions derived from the rheumatic disease use case to evaluate the OHMI ontology. For this purpose, we used the Simple Protocol And Resource Description Framework (RDF) Query Language (SPARQL) and Description Logic (DL) languages. SPARQL is a query language that retrieves data stored in the RDF format [26]. SPARQL queries were performed using the Ontobee's SPARQL endpoint (http://www. ontobee.org/sparql) [25]. The SPARQL scripts are provided in the OHMI GitHub (https://raw.githubusercontent.com/ OHMI-ontology/OHMI/master/docs/SPARQL\%20scripts.

txt). DL queries were performed using the Protégé 5.0 (beta 15) DL Query plugin as described in the Results section.

\section{Ontology access and license}

OHMI is an open source project maintained through https://github.com/ohmi-ontology. The source code, including development and release versions, is available at https://github.com/ohmi-ontology/OHMI. OHMI is released under a Creative Commons 4.0 License. It has been accepted as an OBO library ontology (http://obofoundry.org/ontology/ohmi.html) and deposited in the Ontobee ontology server [25] at http://www.ontobee.
org/ontology/OHMI, and in BioPortal [27] at https:// bioportal.bioontology.org/ontologies/OHMI. Ontobee is the default server for dereferencing OHMI terms.

\section{Results}

\section{OHMI ontology design and upper-level structure}

Figure 1 shows selected upper-level terms and branches of the OHMI hierarchy. Instead of coding everything from scratch, we imported and aligned related terms from existing reference ontologies in the OBO Library, including BFO, NCBITaxon, ENVO, UBERON, OBI, and the Information Artifact Ontology (IAO) [10].

The class OHMI: microbiome is defined as a subclass of ENVO:biome. The latter is defined as follows:

biome $=$ def. an ecosystem to which resident ecological communities have evolved adaptations.

OHMI then defines microbiome as follows:

microbiome $=$ def. a biome that consists of a collection of microorganisms (i.e., microbiota) and the surrounding environment where the microorganisms reside and have evolved adaptations.

OHMI further defines the term 'microbiota' as a subclass of the term 'collection of organisms' in the Population and Community Ontology (PCO):

microbiota $=$ def. a collection of microbial organisms that reside in a particular environment.

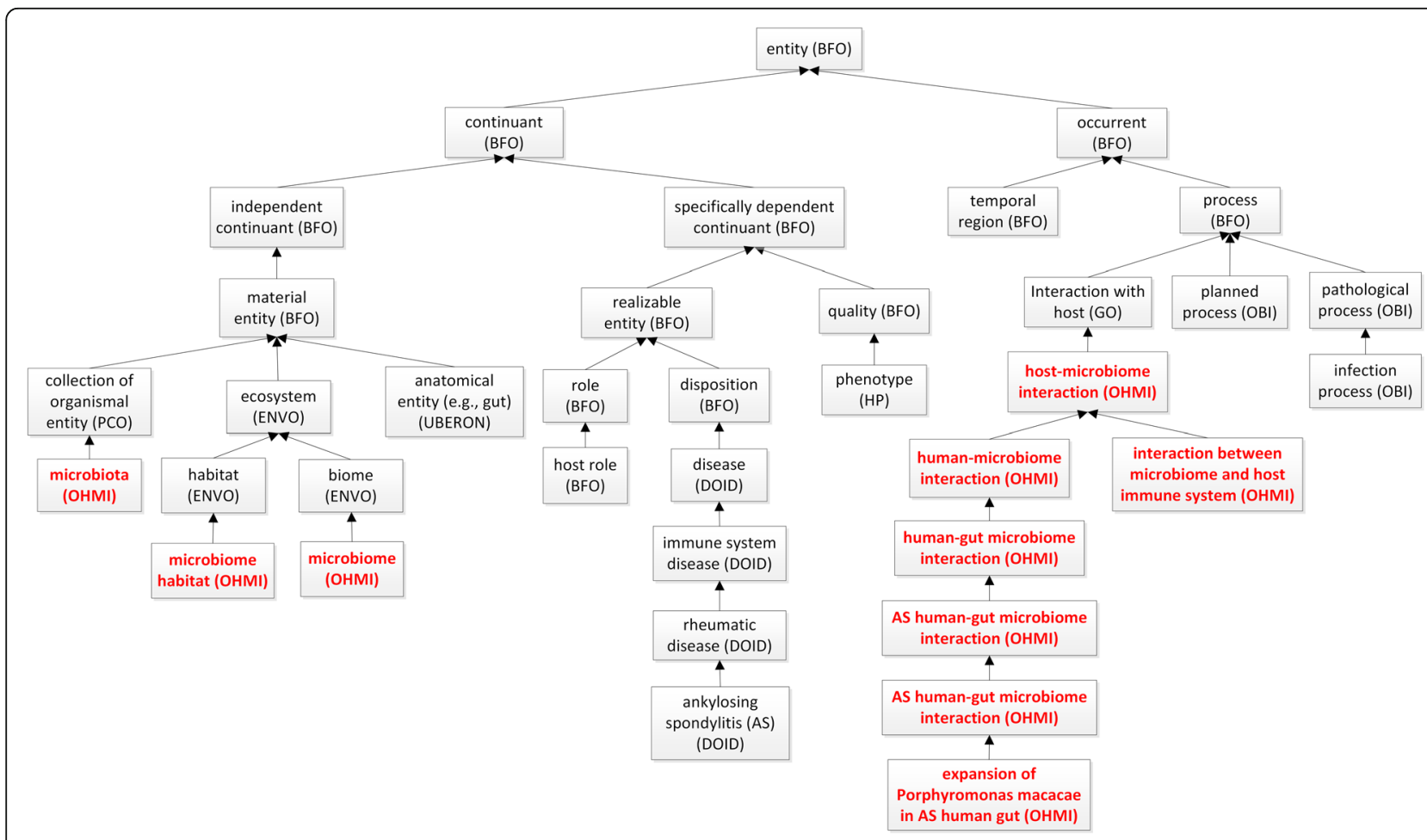

Fig. 1 Selected upper level terms and hierarchy of OHMI. OHMI terms are marked by red labels. The full names of listed ontologies are provided in the list of abbreviations at the end of this paper 
To define the 'host' class in OHMI, we first of all define the host role, which is a BFO:role borne by an entity when one or more further entities are spatially located in its interior. An OHMI:host is then an organism that bears a host role in relation to some microbiome.

The basic design pattern of OHMI is illustrated in Fig. 2. An ontology design pattern is a general pattern to solve a recurrent modeling problem in ontology development by providing scalable and robust representations of entities and entity relations of a certain sort [23]. Terms from the Relation Ontology (RO) [28] have been used to represent OHMI assertions and to formulate corresponding definitions. Specifically, a host-microbiome interaction (HMI) is defined as follows:

host-microbiome interaction $=$ def. an interaction that occurs between a microbiome and its host.

with a logically equivalent class definition as follows:

host-microbiome interaction: interaction and ('has participant' some host) and ('has participant' some microbiome).

Each HMI occurs in some specific anatomic entity (for example the gut) located in the host organism. This host organism may in addition have a disease - a phenomenon that is illustrated by the representation of a general HMI pattern in patients with ankylosing spondylitis (AS) (Fig. 2). In this example, 'AS human-gut microbiota interaction' is a HMI in which the host is a human with AS, while 'gut' is the anatomic entity where the microbiota resides. The expansion of Porphyromonas macacae in AS human gut' is an 'AS human-gut microbiota interaction' in which the size of the population of Porphyromonas macacae is increased (Fig. 2).

As of September 9, 2019, OHMI contains 1238 terms, including 1020 classes, and 128 object properties. OHMI includes 481 OHMI-specific classes and properties with the "OHMI_" prefix, which are new ontology terms not covered in any other OBO Foundry ontologies. More detailed and updated OHMI statistics can be found at the Ontobee statistics page at: http://www.ontobee.org/ontostat/OHMI.

\section{Systematic collection and representation of rheumatic disease-related HMI knowledge}

As a major use case, we systematically collected and annotated the peer-reviewed results of studies of HMI related to rheumatic diseases. Rheumatic diseases are characterized by inflammation of connective tissues, most commonly the joints, but also the tendons, ligaments, bones, muscles, and even solid organs. Our use case study focused on the most common rheumatic diseases, including AS, enthesitis-related arthritis (ERA), gout, psoriatic arthritis (PsA), RA, and systemic lupus erythematosus (SLE), which affect approximately $1 \%$ of the global human population. RA is a common rheumatic

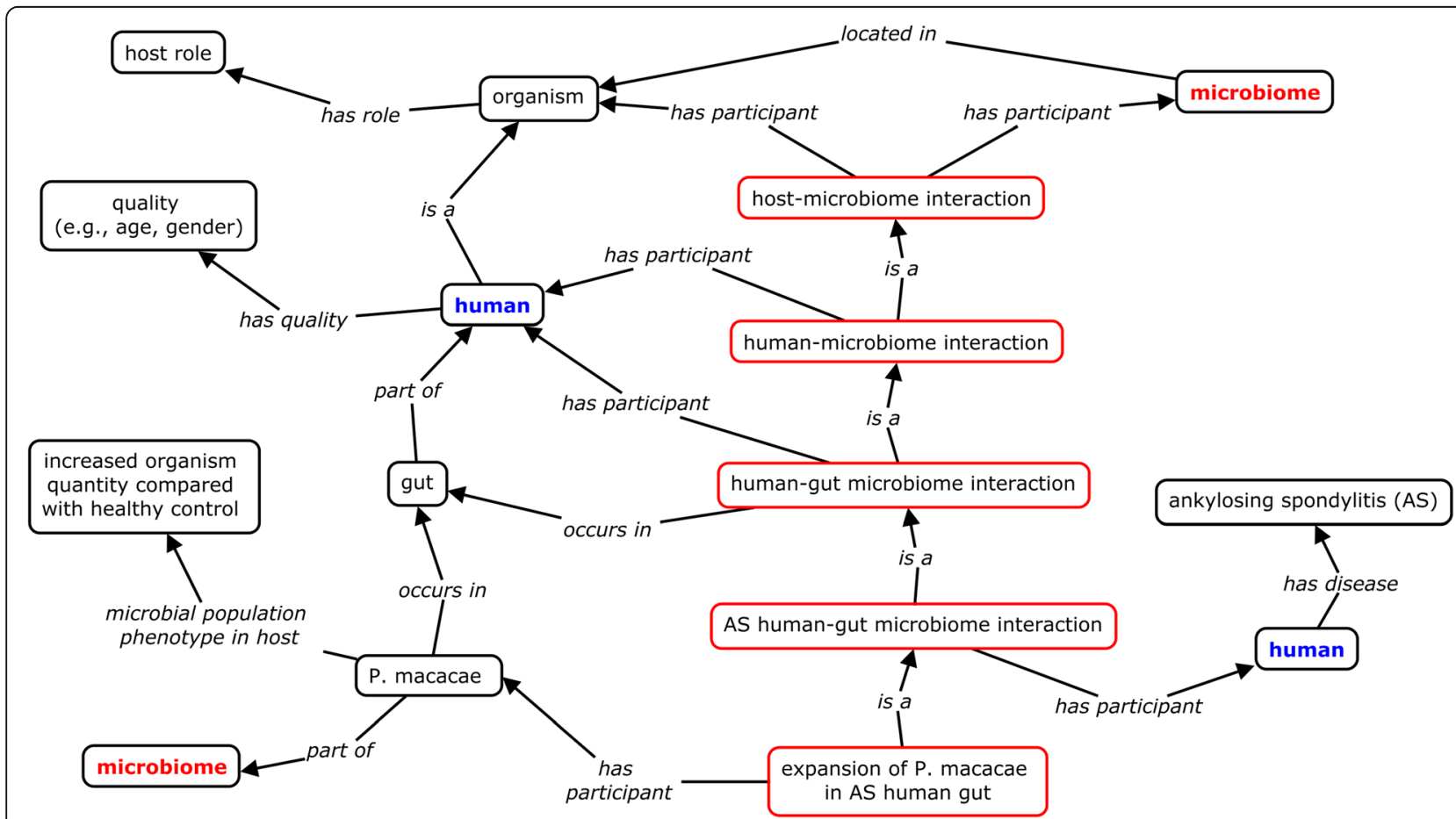

Fig. 2 Illustration of OHMl ontology design pattern for representing host-microbiome interactions. The red box represents different levels of hostmicrobiome interactions. A specific example is the OHMI representation of a human-microbiome interaction in which the human host has the disease ankylosing spondylitis (AS). The human and microbiome classes are duplicated in this figure for clarity. Note that not every organism has the 'host role', and the role is here assigned to a host organism only in the case of host-microbiome interactions 
disease characterized by persistent synovitis, systemic inflammation, and autoantibodies [29]. Many studies have found close associations between rheumatic diseases and HMI [30-33]. Specifically, the gastrointestinal microbiome and its homeostasis are altered in patients with autoimmune and inflammatory rheumatic diseases such as RA [33, 34]. A significant amount of research on the role of the microbiome in autoimmunity has focused primarily on RA [35].

To better understand the relations among rheumatic diseases and microbiomes, we performed a meta-analysis of such relations from relevant literature. In total, from 52 papers (Additional file 2), we found references to 138 bacteria and fungus from the gut, oral cavity, skin, and airway that are associated with the six rheumatic diseases listed above. As an example, the review article by Rosenbaum and Asquith [36] described how microbiome components such as Prevotella copri, Porphyromonas gingivalis, and Collinsella are expanded or depleted in anatomical locations such as the gut, mouth, lung, and skin in the patients of rheumatic diseases, and the possible underlying mechanisms. These microberheumatic disease associations were represented in the OHMI using the design pattern described above (Fig. 2). Our meta-analysis identified increased or decreased microbe populations in patients with different types of rheumatic diseases (Additional file 2).

OHMI can also represent specific types of bacteria that are increased in population size in the intestinal microbiota in at least two rheumatic diseases compared to healthy control (Fig. 3). Fifteen different bacterial categories that are enriched in human patients of different rheumatic diseases (Fig. 3a, Additional file 2). Interestingly the phylum Bacteroidetes includes 10 out of the 15 bacterial species increased in human rheumatic patients. In comparison, only five bacterial categories are decreased in human patients with rheumatic diseases (Fig. 3b). Lachnospiraceae is increased in AS and SLE patients [37, 38] (Fig. 3a), but decreased in ERA and gout patients [39, 40] (Fig. 3b). Coprococcus and Pseudobutyrivibrio, two bacteria types within Lachnospiraceae, were

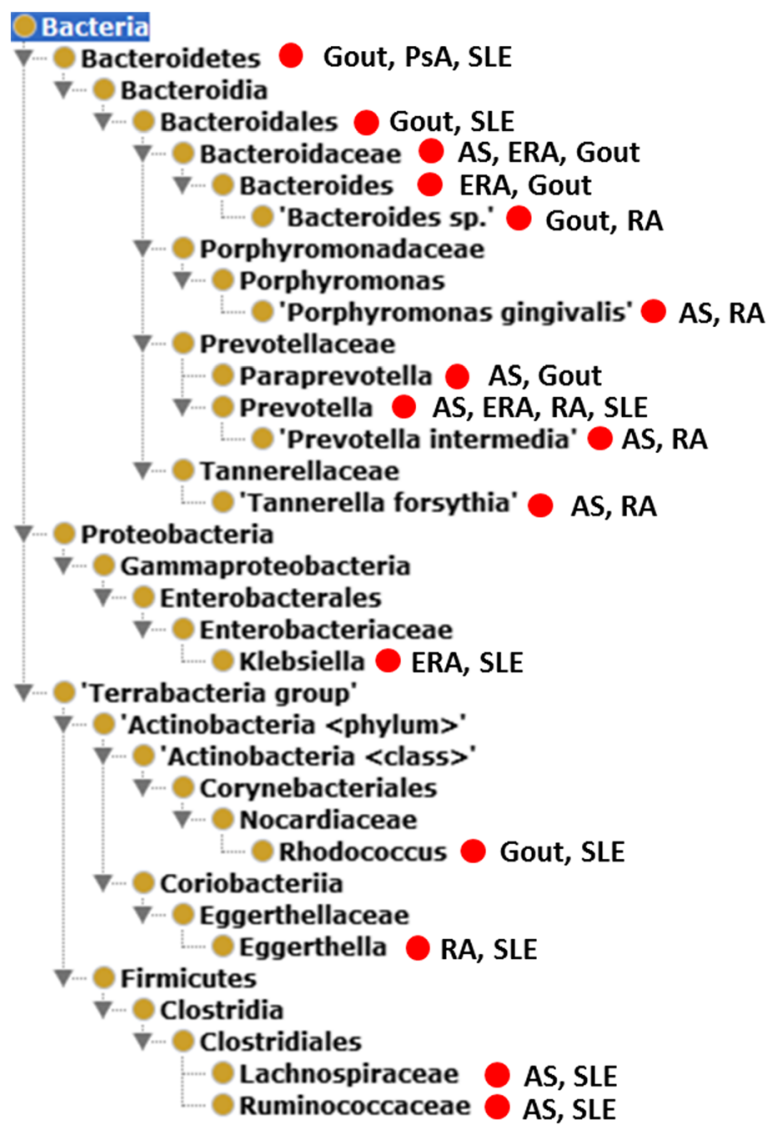

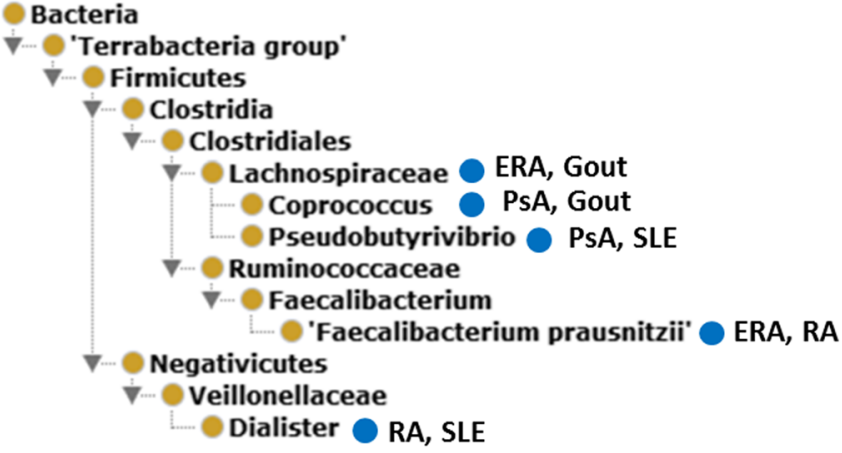

(b)

(a)

Fig. 3 Ontological representation of the bacteria populations increased in the guts of patients with at least two different rheumatic diseases as compared with healthy controls. (a) Bacterial population increased in patient guts. (b) Bacterial population decreased in patient guts. Many increased and decreased bacterial populations are within the same genus. The red and blue circles represent increased and decreased profiles, respectively. Taxonomy terms without circle and label are used to generate ontological hierarchies 
also found to be depleted in rheumatic diseases PsA [41] and gout [39, 41], and SLE [42].

OHMI representation of key terms in an HMI investigation A good representative example of the many studies on host-microbiome interactions is the study of the interaction between rheumatic arthritis and human microbiome documented in [43]. In this study, samples were collected from patients before or after antibiotic treatment, and the diversity and composition of the respective microbiome constitutions were identified via $16 \mathrm{~S}$ rRNA gene sequencing. This study set out to address three key HMI questions: (i) how do healthy and RA patients differ in the compositions of their microbiome? (ii) does the composition of the microbiome in RA patients shift as a result of antibiotic treatment? and (iii) are distinct clinical parameters in RA patients (e.g., autoantibody profiles) associated with distinct microbial community profiles? Analogues of these three questions appear in many microbiome studies across human, animal and environmental disciplines.

To annotate a HMI study as illustrated above [43], we draw on the general OHMI design pattern (Fig. 4). Basically, an HMI investigation involves the collection and analysis of microbiome specimens from an anatomical location in a host organism. The host organism will have a certain demographic profile (sex, age and so forth). It may be either a patient with a specific disease or a healthy control. It may be treated with different interventions, and the samples can be collected at different time points subsequent to the medical intervention. The processed samples are assayed, generating different datasets. Conclusions can then be drawn on the basis of the statistical analysis of these datasets.
The investigation of OHMI host-microbiome interactions involves the representation of various metadata (i.e., "data about data") types. To standardize these, we reference different resources, starting with the Minimum Information about a Genome Sequence (MIGS) [44] and about any (' $x$ ') Sequence (MIxS) checklists [45]. While the latter specifies the variables and the information required to describe a genome sequence or any sequence, they do not cover all the information about HMIs and they do not specifically use ontologies. The metadata types documented in these checklist resources were complemented by specific metadata types used in the studies recorded in the peerreviewed publications such as $[43,46,47]$. These metadata types can be matched to entities defined in ontology.

Table 1 lists a representative minimal checklist of entities that are important for representing an HMI investigation. These terms cover various areas such as host, microbiome sample extraction and analysis, host treatment, and microbiome profile changes. These terms are matched to the metadata types collected for HMI investigation studies. OHMI has imported many corresponding terms from existing ontologies. We also generated many OHMI-specific terms for representation of host-pathogen interactions (Table 1). As a result, OHMI allows us to standardize the representation of a broad range of HMI experiments, and to do this in a way that leads to more advanced integrative data/metadata analyses of such studies, including analyses relating to sequence and other data.

\section{Addressing competency questions using OHMI}

In addition to the above-mentioned use case of rheumatic disease-associated HMI representation, we also applied OHMI to address several real-life competency questions in additional use cases.

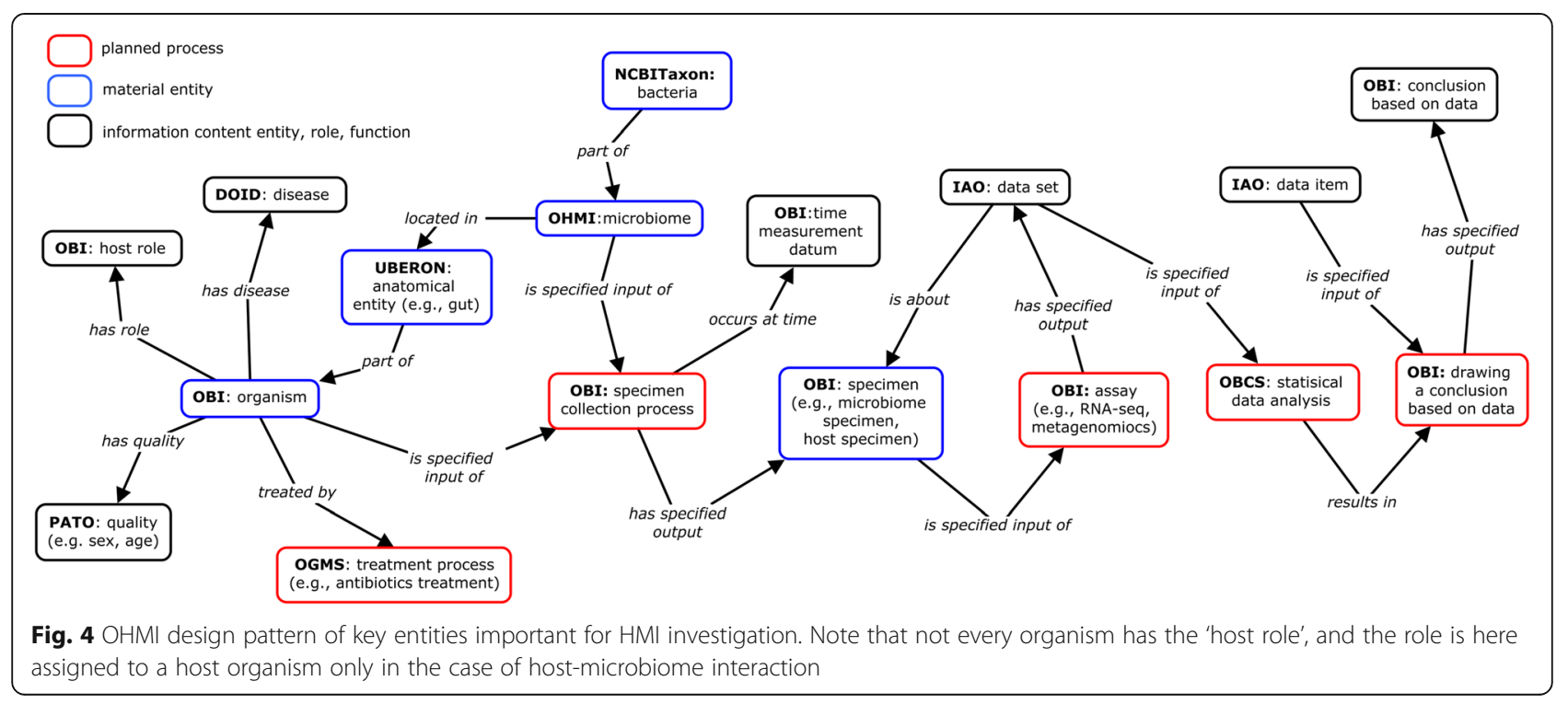


Table 1 Selected OHMl entities important for HMl investigation

\begin{tabular}{|c|c|c|}
\hline Topics & Example terms & Ontology \\
\hline Host & $\begin{array}{l}\text { host organism (e.g., human, rat); age, biological sex; } \\
\text { disease (e.g., RA, diarrhea); phenotype (e.g., obesity, diarrhea); host anatomical entity (e.g., mouth, } \\
\text { stomach); drug product; dysbiosis }\end{array}$ & $\begin{array}{l}\text { NCBITaxOn PATO } \\
\text { DOID MPO, HPO, ... } \\
\text { UBERON DRON } \\
\text { OHMI }\end{array}$ \\
\hline Microbe & $\begin{array}{l}\text { microbial taxonomy at various levels (e.g., E. coli); } \\
\text { species abundance, microbial diversity, microbial } \\
\text { genome }\end{array}$ & $\begin{array}{l}\text { NCBITaxon } \\
\text { OHMl }\end{array}$ \\
\hline Environment conditions & $\begin{array}{l}\text { environment (e.g., dwelling, wild field); } \\
\text { metabolite (e.g., iron, zinc and arginine), nutrition, ... }\end{array}$ & ENVO CHEBI \\
\hline Sample collection & $\begin{array}{l}\text { collection date/time, collection method, device; } \\
\text { geographic location }\end{array}$ & $\mathrm{OBI} G A Z$ \\
\hline HMI samples & $\begin{array}{l}\text { sample from host, e.g., gut, oral, saliva; } \\
\text { sample from environment, e.g., soil, table surface }\end{array}$ & OBI ENVO \\
\hline Assays & RNA-seq, genome sequencing & $\mathrm{OBI}$ \\
\hline Statistical analyses & $\begin{array}{l}\text { ANOVA, t-test, Wilcoxon rank-sum test, MLG-based } \\
\text { classifier, KEGG analysis, metagenomic sequencing } \\
\text { data, } p \text {-value }\end{array}$ & OBCS \\
\hline HMI results & $\begin{array}{l}\text { relative abundance of microbe in host, a-diversity, } \\
\text { differentially enriched bacterium (or gene) marker } \\
\text { for dysbiosis/disease, overgrowth vs. depletion } \\
\text { (or reduced growth); microbiome restoration by } \\
\text { treatment (e.g., antibiotics, DMARD) }\end{array}$ & OHMI (4-5) \\
\hline
\end{tabular}

The column 'Ontology' represents the source ontology in which the example terms are defined. All the terms are defined either in OHMI or imported from other ontologies to $\mathrm{OHMI}$

Competency question 1: What are the human diseases for which a bacterium or bacterial group (for example, E. coli) is expanded in population size in the microbiome?

A commonly asked competency question relates to the identification of the microbiome components that are increased in humans with a specific disease as compared with healthy control subjects. For example, Porphyromonas macacae is expanded in the gut of AS patients, which we represent by means of the term 'expansion of Porphyromonas macacae in AS human gut' (Fig. 2). This OHMI relationship between the HMI and the bacterium Porphyromonas macacae is here represented as:

'expansion of Porphyromonas macacae in AS human gut': 'has microbe expanded in diseased host' some 'Porphyromonas macacae'.

The relational expression 'has microbe expanded in diseased host' is an object property that represents the relation between a host-microbiome interaction (HMI) and a microbe, where the population of the microbe is expanded in a diseased host as compared to a healthy host control. As illustrated in the above example, the domain of the relation is a HMI process, and the range is a microbe such as a bacterium. This specific relation is formulated in natural language by means of several different expressions. The inclusion of such a specific relation in OHMI provides a single target for the corresponding annotations which represents a direct logical linkage between a disease-specific HMI and a microbe. OHMI thereby supports efficient knowledge querying and analysis also where we take natural-language inputs as our starting point.

We can use the relationship "has microbe expanded in diseased host' to represent the disease-associated HMIs related to each specific bacterium or bacterium group. For example, such a representation method can be used to identify all the E. coli-associated human diseases investigated in [48-51] on the basis of a single DL query, using either Protégé (Fig. 5a) or the Ontobee SPARQL endpoint (Fig. 5). The same outcome was achieved using either approach. The results indicate that E. coli and four specific $E$. coli strains were found in rheumatic arthritis, gout, and colorectal cancer.

\section{Competency question 2: What microorganisms are expanded or depleted in subjects with a specific disease (for example $R A$ ) relative to health controls?}

OHMI records manually mined knowledge relating to many types of microorganisms that are increased or decreased in population size in patients with a specific disease relative to healthy controls. This knowledge is often obtained by text-mining all the papers referenced in the literature reports of well-controlled epidemiological studies. OHMI represents this knowledge in two ways. First, it creates a logically well-defined representation of a specific HMI in association with specific disease and microbiome information, as exemplified in Fig. 2 and also shown below: 


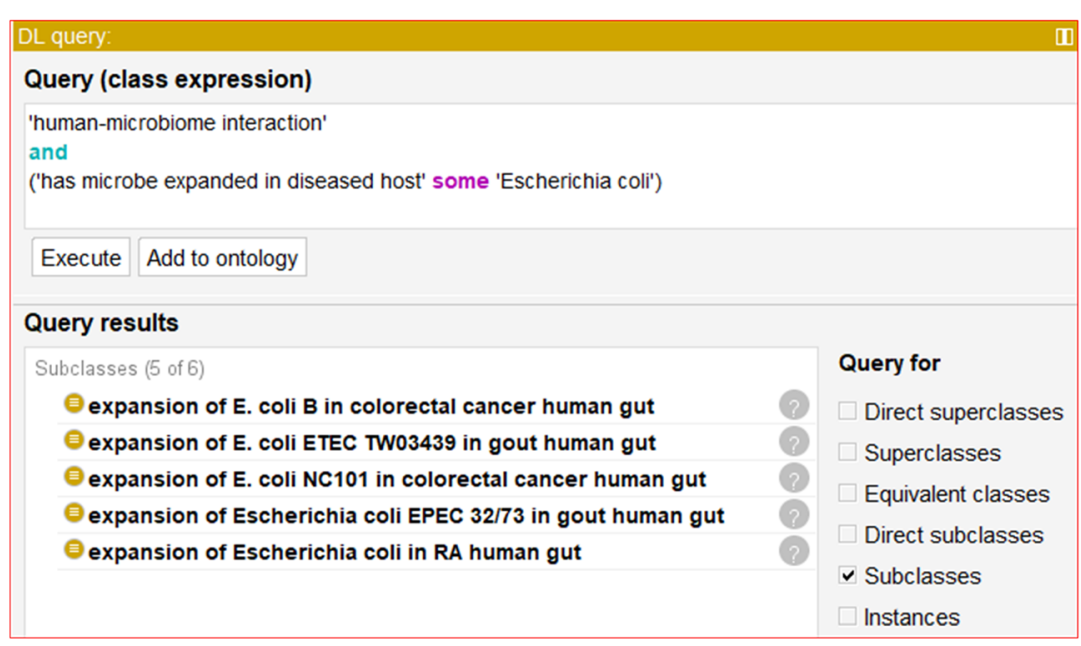

(a)

\# Goal: find E. coli-associated human-microbiome interactions

PREFIX Escherichia: 〈http://purl.obolibrary.org/obo/NCBITaxon_561>

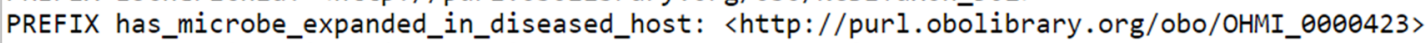

PREFIX human_microbiome_interaction: 〈http://purl.obolibrary.org/obo/OHMI_0000019>

SELECT distinct (STR(?microbe_label) AS ?microbe_label) (STR(?interaction_label) AS ?interaction_label)

FROM 〈http://purl.obolibrary.org/obo/merged/OHMI〉

WHERE

\{

?microbe rdfs:label ?microbe_label.

?interaction rdfs:label ?interaction_label .

?microbe rdfs: subclassof Escherichia: option (transitive).

?interaction rdfs:subClassof human_microbiome_interaction: option (transitive).

?interaction rdfs: subclassof ?in_restriction .

\}

?in_restriction owl:onProperty häs_microbe_expanded_in_diseased_host:; owl:someValuesFrom ?microbe .

\begin{tabular}{|l|l|l|l|l|}
\hline Output format & Table & V Max Rows $10 \quad$ V \\
\hline Run Query & Reset \\
\hline
\end{tabular}

Result Raw Request/Permalinks Raw Response

\begin{tabular}{|l|l|}
\hline \multicolumn{1}{|c|}{ microbe_label } & \multicolumn{1}{c|}{ interaction_label } \\
\hline Escherichia coli & expansion of Escherichia coli in RA human gut \\
\hline Escherichia coli EPEC 32/73 & expansion of Escherichia coli EPEC 32/73 in gout human gut \\
\hline Escherichia coli ETEC TW03439 & expansion of E. coli ETEC TW03439 in gout human gut \\
\hline Escherichia coli NC101 & expansion of E. coli NC101 in colorectal cancer human gut \\
\hline Escherichia coli B & expansion of E. coli B in colorectal cancer human gut \\
\hline
\end{tabular}

(b)

Fig. 5 Query of diseases associated with increased E. coli in human gut. (a) DL query based on the host-pathogen interaction classifications; (b) SPARQL query based on the linkage from organism to disease. The SPARQL query was conducted using the Ontobee SPARQL endpoint (http://www.ontobee.org/sparql)

'expansion of Porphyromonas macacae in AS human gut': 'has microbe expanded in diseased host' some ('Porphyromonas macacae' and ('located in' some ('lower digestive tract' and ('part of' some ('Homo sapiens' and ('has disease' some 'ankylosing spondylitis')))))).

The above is a class definition formulated using logical equivalence. It thus provides necessary and sufficient conditions for the specific HMI class to be exemplified.
Using this type of definition, we can specify the link between the microbe P. macacae and the disease ankylosing spondylitis (AS). This style of logical definition can serve as a basis for both DL and SPARQL queries. However, since the logical definition includes four relations and is quite complex, it is difficult to write efficient query scripts for queries of this sort.

To solve this problem, OHMI provides shortcut relations to link an organism directly to a disease, for 
example, 'has microbe depleted in gut of human with disease', which is used as follows:

\section{Prevotella:}

'microbe susceptibly depleted in gut of human with disease' some 'rheumatoid arthritis'.

From this we can easily generate DL or SPARQL query scripts. For example, using a SPARQL query using the above relation, we quickly identified 45 distinct bacterial species that are associated with RA through six different relationships (Additional file 1: Figure S1). These 45 species can be organized using an ontological hierarchy (Fig. 6), which reveals several interesting phenomena. For example, both Lactobacillus sp. and Lactobacillus salivariusc are increased in RA patients, the former in the gut, the latter in the oral cavity. On the other hand, five bacteria under Betaproteobacteria were all depleted in RA patients. Among them, three Neisseriaceae bacterial groups (Eikenella, Kingella, and Neisseria spp.) are all decreased in the oral cavity in the RA patients, two Burkholderiales groups (Burkholderia and Sutterella wadsorthensis) are depleted in the gut or respiratory airway of the RA patients. Interestingly, Bifidobacterium bifidum is decreased in the gut of RA patients; however, Bifidobacterium dentium is increased (Fig. 6). These findings merit further investigation to identify the underlying mechanisms associated with each of these phenomena.

\section{Discussion}

This paper introduces the development and application of a community-driven ontology to represent HMIs. OHMI standardizes HMI-related terms and the relations among them. The top level hierarchical structure and general OHMI design pattern are described, followed by descriptions of uses of OHMI with examples and competency questions. OHMI was developed by following best practices [8] and recommended strategies of ontology reuse [7] and design pattern-based development [23]. OHMI seamlessly integrates related terms from existing ontologies with terms specifically related to HMIs. Our use cases demonstrate that OHMI is useful in HMI knowledge representation, data and metadata standardization, and information query and analysis.

OHMI provides a consistent and hierarchical representation of the known HMIs, where the individual microbes, anatomic locations, host species, and host qualities are also represented using the ontology. As shown in the rheumatic disease use case, we were able to identify those expanded and depleted microbes associated with rheumatic diseases. The ontological classification of expanded microbes suggests possible virulent factors and potential pathogenesis in rheumatic diseases, whereas queries identifying depleted bacteria may identify potential candidates for treatment with biological agents [52].
OHMI development is a community effort. For example, OHMI was presented at the Sixth Annual Workshop of the Clinical and Translational Science Ontology Group held in October of 2017 in Ann Arbor, Michigan, USA [53]. The focus of this meeting was to introduce different microbiology-related ontologies, including OHMI, in order to better understand the type of standardization needed to facilitate data integration. There was a consensus that the OHMI and other microbiology-related ontologies should be developed and used by the community to support not only data and knowledge integration but also the design of experimental studies that would be reproducible.

Although the knowledge represented in OHMI is commonly obtained from review of published papers, we can also analyze raw data and generate new information about microbiome and relation examples from an existing database. MicrobiomeDB (http://microbiomedb.org) is a web-based database and systems biology platform for integrating, mining and analyzing data from microbiome experiments [46]. We used the platform to search, analyze and compare microbiome profiles under different conditions. For example, MicrobiomeDB collected over 1000 samples used for study of diarrheal disease and the microbiome in children [54, 55]. Using the dataset collected in MicrobiomeDB and the data analysis tool in the database, we found that Prevotella is significantly lower in diarrheal patients compared to controls; however, for unclassified Proteobacteria and Natronobacillus bacteria the converse was true (Fig. 7a). In addition to the disease condition, age was also found to be a major factor. Our MicrobiomeDB analysis found that the amount of $E$. coli was low in the healthy state in the guts of infants compared to children, but was significantly higher in the infant gut during diarrhea than in the healthy state. The results obtained from analysis of the large MicrobiomeDB dataset can be semantically represented using OHMI (Fig. 7b). This permits the querying of MicrobiomeDB results together with other information drawn from peer-reviewed results. Future directions will include more analysis and representation of MicrobiomeDB data in OHMI in order to improve representation and understanding of HMI mechanisms.

Reproducibility of both experiments and data analyses depends on consistent procedures employed across different settings. OHMI harmonizes the metadata representation of the interactions between microbiomes and hosts along with associated biological processes, supporting the integration and systems-level analysis of HMI data produced by different laboratories and institutions. To support reproducibility and advanced analysis, we plan to work with the MicrobiomeDB project to support ontology-based data standardization, browsing, and advanced analyses. Specifically, MicrobiomeDB will use OHMI terms and metadata 


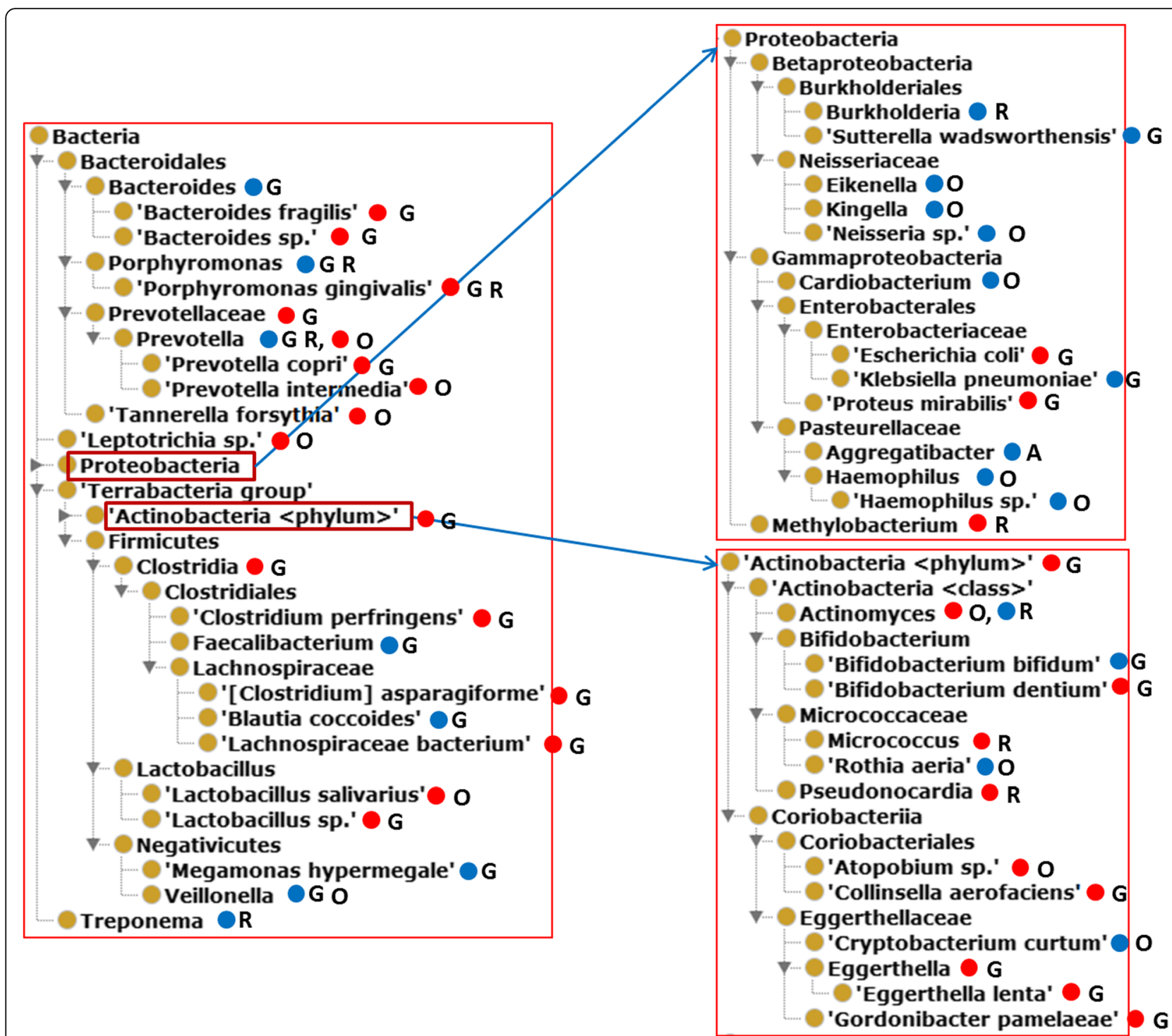

Fig. 6 The hierarchy of microbes associated with RA and their profiles. The red and blue circles represent the increased and decreased profiles, respectively. Labeled letters represent locations as follows: $\mathrm{G}$ - human gut, $\mathrm{O}$ - human oral cavity; R - human respiratory airway. Those taxonomy terms without circle and label are used only to generate the hierarchy

types to standardize the currently still highly heterogeneous MicrobiomeDB data. The information captured in OHMI can also provide prior knowledge that can be used to enhance queries and analyses of MicrobiomeDB data. One such strategy is to allow users who have identified differentially abundant taxa (using the existing differential abundance app) to leverage OHMI to ask whether the identified list of differentially abundant taxa is enriched for any disease processes or interactions. Such an approach is similar to how the Gene Ontology (GO) has been used to support data analysis, by providing prior knowledge relating to the roles of given genes in realizing given functions, knowledge which can then be used to support gene enrichment and other data analysis [56]. Such a strategy has its advantages over alternatives such as simply using relational database representations, since the ontology-based approach supports better standardization, flexibility, interoperability, machine interpretation, and extensible tool development.

A newly funded project is to apply OHMI to study the host-microbe interactions related to gastric cancer. Gastric cancer $(\mathrm{GC})$ is the fifth most prevalent malignancy and the third leading cause of cancer death worldwide. Almost half of new cases occur in China, and it is the second leading cause of cancer death in China. The strongest risk factor for gastric cancer is chronic Helicobacter pylori infection. People with an $H$. pylori infection have a roughly six-fold greater risk of developing gastric cancer than uninfected people. However, not all people 


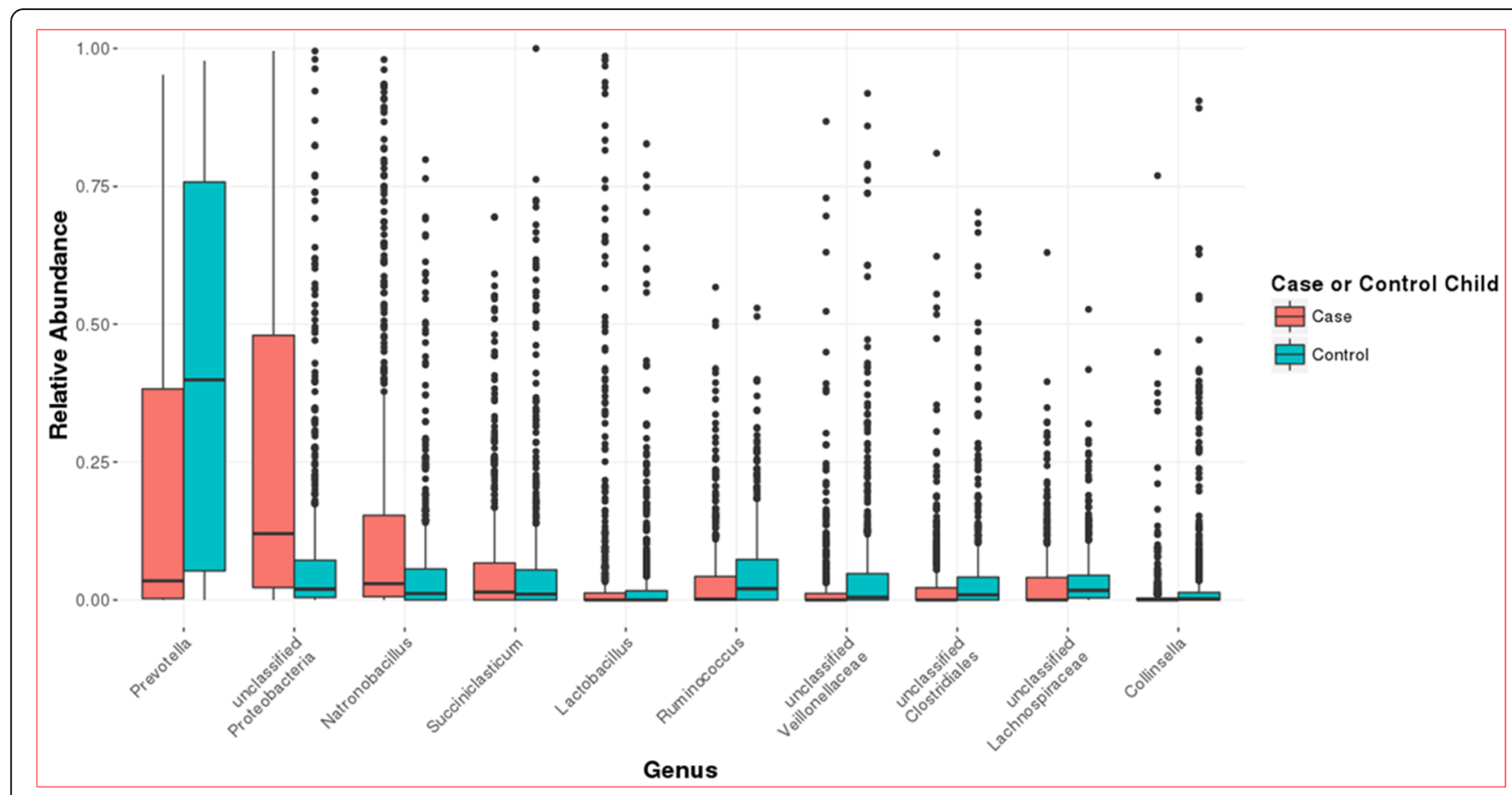

(a)
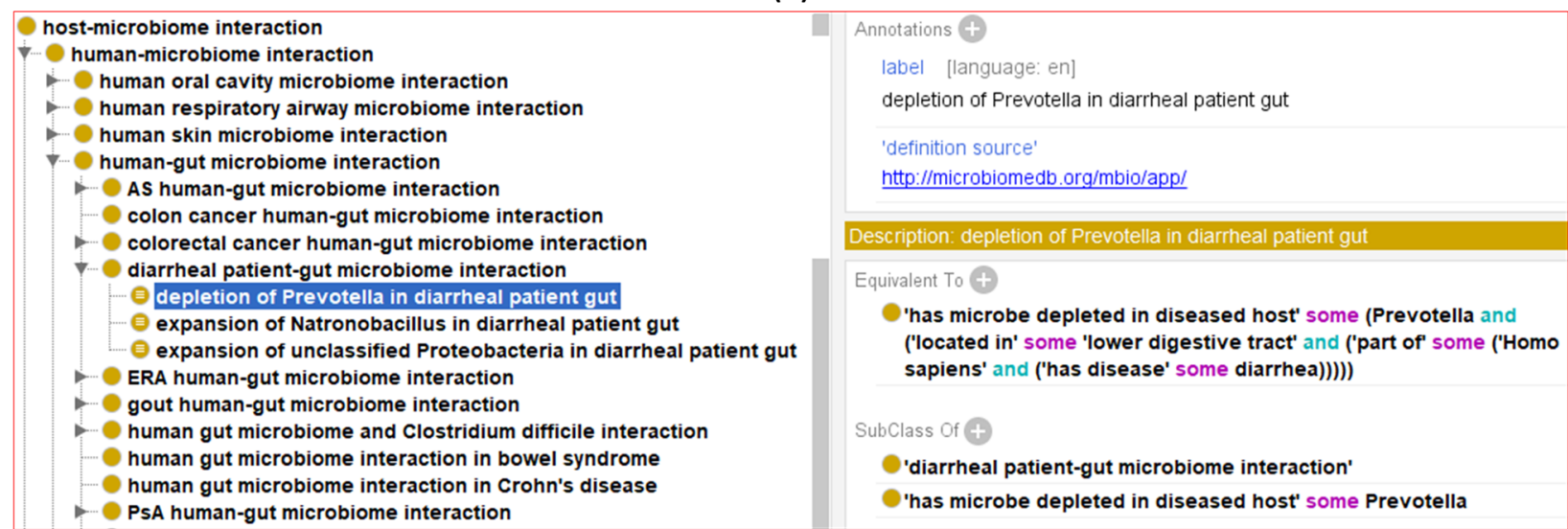

(b)

Fig. 7 Data mining and ontology representation of microbiome profiles at different species level between diarrhea and health controls. (a) MicrobiomeDB data mining. (b) OHMI representation of the results

infected with $H$. pylori have gastric cancer, suggesting that there are more factors and mechanisms involved in gastric carcinogenesis which are not yet understood. Comparative bacterial genomic analysis in patients with or without gastric cancer allows gene-level study of host-microbiome interactions as it relates to gastric carcinogenesis in humans. Clinical trials together with multi-omics studies are being performed to further explore the mechanisms of host-microbiome interactions leading to gastric cancer.

OHMI is an ongoing project. Future work includes expansion of OHMI to cover more diseases such as obesity and inflammatory bowel disease. More molecular and cellular entities and processes will be included to better understand HMI mechanisms. Such host-specific microbiome profiles may serve as a biological marker for specific host types or specific health conditions. Various types of biological conditions (for example associated with patient age, biological sex, genetic makeup, and environment) may affect the outcomes of HMIs and will be systematically studied. Thus, one important outcome of OHMI is to identify opportunities to collect microbiome data related to pervasive exposures that cut across multiple disease states (for example tobacco smoking and Western versus traditional diets) and which were not captured by the Human Microbiome project. OHMI is expected to become an ontology-based 
interoperable knowledge base of host-microbiome interactions, which can be used to address many technical challenges in constructing microbiome-disease association knowledge bases [57] and thereby help to solve fundamental scientific questions. We also welcome researchers interested in the topic to participate in the community-based OHMI development and its application to other use cases.

\section{Conclusion}

OHMI ontologically represents entities of various types associated with HMIs and the relations among such entities. Our use cases demonstrate how OHMI could be used as a canonical platform for HMI knowledge representation, metadata standardization, semantic querying and data analysis.

\section{Supplementary information}

Supplementary information accompanies this paper at https://doi.org/10. 1186/s13326-019-0217-1.

Additional file 1: Figure S1. SPARQL query for all microbes associated with RA and their relations. The query was conducted using the Ontobee SPARQL endpoint

Additional file 2: Manually annotated rheumatic disease HMI data from the literature. The annotated information is represented in $\mathrm{OHMl}$

\section{Abbreviations}

AS: Ankylosing spondylitis; BFO: Basic Formal Ontology; ChEBl: Chemical Entities of Biological Interest; DL query: Description Logics query; DMARD: Disease-modifying anti-rheumatic drug; DOID: Disease Ontology; DRON: Drug Ontology; ERA: Enthesitis-related arthritis; GO: Gene Ontology; IAO: Information Artifact Ontology; MicO: Ontology of Prokaryotic Phenotypic and Metabolic Characters; MPO: Mammalian Phenotype Ontology; NCBITaxon: NCBI organismal classification; OBCS: Ontology of Biological and Clinical Statistics; OBI: Ontology for Biomedical Investigations; OBO: The Open Biological and Biomedical Ontologies; OHMI: Ontology of Host-Microbiome Interactions; OMP: Ontology for Microbial Phenotypes; OWL: Web Ontology Language; PATO: Phenotypic Quality Ontology; PR: Protein Ontology; PsA: Psoriatic arthritis; RA: Rheumatoid arthritis; RDF: Resource Description Framework; SLE: Systemic lupus erythematosus; SPARQL: SPARQL Protocol and RDF Query Language; UBERON: Uberon multi-species anatomy ontology

\section{Acknowledgements}

We acknowledge wide-ranging discussions and feedback from Drs. Pier Luigi Buttigieg, Ramona L. Walls, Christopher J. Mungall, William R. Hogan, Amanda Hicks, and other experts in the host-microbiome interaction community and the biomedical ontology community. We also appreciate the support by Dr. Amanda Hicks, Dr. William R. Hogan, Dr. George Mashour, Dr. Robert C. Dysko, Mr. Jamie Racklyeft, Ms. Veronica Rieberger, and Ms. Teresa Fracala for their support on the Sixth Annual Workshop of the Clinical and Translational Science Ontology Group, Ann Abor, MI, USA, on October 25-26, 2017.

\section{Authors' contributions}

$\mathrm{YH}$ : Primary OHMI developer, pattern design, use case analysis, and first manuscript drafting. HW: Rheumatic disease use case knowledge extraction and $\mathrm{OHMI}$ representation, data analysis and result interpretation. JZ: active $\mathrm{OHMI}$ developer, pattern design, and use case design. DP: MicrobiomeDB use case design and implementation. AMM: active $\mathrm{OHMI}$ developer and use case analysis. HY: rheumatic disease use case annotation, OHMI design and development. $\mathrm{KL}$ and JW: active OHMI developers, gastric cancer use case study. JLC: respiratory use case annotation. TP: host response modeling in OHMI. BS: high level ontology alignment. AVA: use case study. JSO: active $\mathrm{OHMI}$ developer; use case design and analysis, and OHMI meeting organization. All contributed to manuscript preparation and revision. All authors read and approved the final manuscript.

\section{Funding}

This project was supported by grants: 101 CX000911, UL1TR001450, UL1TR001412, R01LM012517, R21TR002513, R01GM080646, and U54CA210962. It was also supported by the Medical University of South Carolina (MUSC) College of Medicine Enhancing Team Science (COMETS) award, the University of Michigan Global Reach award, and a grant from the Michigan Medicine-Peking University Health Sciences Center Joint Institute for Clinical and Translational Research (U063430 and BMU2019J1010). We also acknowledge the Michigan Institute for Clinical \& Health Research (MICHR, UL1TR002240) and University of Michigan Unit for Laboratory Animal Medicine (ULAM) for their sponsorship of the Sixth Annual Workshop of the Clinical and Translational Science Ontology Group.

\section{Availability of data and materials}

All data generated or analyzed during this study are included in this published article and Additional files.

Ethics approval and consent to participate Not applicable.

\section{Consent for publication}

Not applicable.

\section{Competing interests}

The authors declare that they have no competing interests.

\section{Author details}

${ }^{1}$ University of Michigan Medical School, Ann Arbor, MI 48109, USA. ²Daqing Branch of Harbin Medical University, Daqing 163319, Heilongjiang, China. ${ }^{3}$ University of Pennsylvania Perelman School of Medicine, Philadelphia, PA 19104, USA. ${ }^{4}$ University of Pennsylvania School of Veterinary Medicine, Philadelphia, PA 19104, USA. ${ }^{5}$ Department of Biostatistics and Bioinformatics, Duke University School of Medicine, Durham, NC 27710, USA. 'People's Hospital of Guizhou Province, Guiyang 550025, Guizhou, China. ${ }^{7}$ School of Public Health, Anhui Medical University, No 81 Meishan Road, Hefei 230032, Anhui, China. ${ }^{8}$ Center for Cancer Bioinformatics, Peking University Cancer Hospital \& Institute, Beijing 100142, China. ${ }^{9}$ Pulmonary \& Critical Care Medicine Section, Medical Service, VA Ann Arbor Healthcare System, Ann Arbor, MI 48105, USA. ${ }^{10}$ University at Buffalo, Buffalo, NY 14260, USA.

${ }^{11}$ Department of Public Health Sciences, Medical University of South Carolina, Charleston, SC 29425, USA.

Received: 9 February 2019 Accepted: 4 December 2019

Published online: 30 December 2019

\section{References}

1. Group NHW, Peterson J, Garges S, Giovanni M, Mclnnes P, Wang L, Schloss JA, Bonazzi V, McEwen JE, Wetterstrand KA, et al. The NIH human microbiome project. Genome Res. 2009;19(12):2317-23.

2. Marchesi JR, Ravel J. The vocabulary of microbiome research: a proposal. Microbiome. 2015:3:31.

3. Sunagawa S, Coelho LP, Chaffron S, Kultima JR, Labadie K, Salazar G, Djahanschiri B, Zeller G, Mende DR, Alberti A, et al. Ocean plankton. Structure and function of the global ocean microbiome. Science. 2015; 348(6237):1261359.

4. Shi N, Li N, Duan X, Niu H. Interaction between the gut microbiome and mucosal immune system. Military Medical Research. 2017:4:14.

5. Rivera-Amill V. The Human Microbiome and the Immune System: An Ever Evolving Understanding. J Clin Cell Immunol. 2014;5(6).

6. Kau AL, Ahern PP, Griffin NW, Goodman AL, Gordon Jl. Human nutrition, the gut microbiome and the immune system. Nature. 2011;474(7351):327-36.

7. Wilkinson MD, Dumontier M, Aalbersberg IJ, Appleton G, Axton M, Baak A, Blomberg N, Boiten JW, da Silva Santos LB, Bourne PE, et al. The FAIR guiding principles for scientific data management and stewardship. Scientific data. 2016;3:160018.

8. Hur J, Ozgur A, He Y. Ontology-based literature mining of E. coli vaccineassociated gene interaction networks. J Biomed Semantics. 2017:8(1):12. 
9. NCBITaxon: An ontology representation of the NCBI organismal taxonomy. [http://obofoundry.org/ontology/ncbitaxon.html]. Accessed 10 Dec 2019.

10. Mungall CJ, Torniai C, Gkoutos GV, Lewis SE, Haendel MA. Uberon, an integrative multi-species anatomy ontology. Genome Biol. 2012;13(1):R5.

11. Buttigieg PL, Morrison N, Smith B, Mungall CJ, Lewis SE, Consortium E. The environment ontology: contextualising biological and biomedical entities. J Biomed Semantics. 2013;4(1):43.

12. Chibucos MC, Zweifel AE, Herrera JC, Meza W, Eslamfam S, Uetz P, Siegele DA, Hu JC, Giglio MG. An ontology for microbial phenotypes. BMC Microbiol. 2014;14:294.

13. Blank CE, Cui H, Moore LR, Walls RL. MicrO: an ontology of phenotypic and metabolic characters, assays, and culture media found in prokaryotic taxonomic descriptions. J Biomed Semantics. 2016;7:18.

14. PATO - Phenotypic Quality Ontology [https://github.com/pato-ontology/pato/]. Accessed 14 Dec 2019.

15. Ashburner M, Ball CA, Blake JA, Botstein D, Butler H, Cherry JM, Davis AP, Dolinski K, Dwight SS, Eppig JT, et al. Gene ontology: tool for the unification of biology. The gene ontology Consortium. Nat Genet. 2000;25(1):25-9.

16. Hastings J, de Matos P, Dekker A, Ennis M, Harsha B, Kale N, Muthukrishnan V, Owen G, Turner S, Williams M, et al. The ChEBI reference database and ontology for biologically relevant chemistry: enhancements for 2013. Nucleic Acids Res. 2013;41(Database issue):D456-63.

17. Natale DA, Arighi CN, Blake JA, Bult CJ, Christie KR, Cowart J, D'Eustachio P, Diehl $A D$, Drabkin HJ, Helfer $O$, et al. Protein ontology: a controlled structured network of protein entities. Nucleic Acids Res. 2014;42(Database issue):D415-21.

18. Bandrowski A, Brinkman R, Brochhausen M, Brush MH, Bug B, Chibucos MC, Clancy K, Courtot M, Derom D, Dumontier M, et al. The ontology for biomedical investigations. PLoS One. 2016;11(4):e0154556.

19. Smith B, Ashburner M, Rosse C, Bard J, Bug W, Ceusters W, Goldberg LJ, Eilbeck K, Ireland A, Mungall CJ, et al. The OBO foundry: coordinated evolution of ontologies to support biomedical data integration. Nat Biotechnol. 2007;25(11):1251-5.

20. He Y, Xiang Z, Zheng J, Lin Y, Overton JA, Ong E. The eXtensible ontology development $(X O D)$ principles and tool implementation to support ontology interoperability. J Biomed Semantics. 2018;9(1):3.

21. Arp R, Smith B, Spear AD. Building ontologies using basic formal ontology. Cambridge, MA, USA: MIT Press; 2015.

22. Xiang Z, Courtot M, Brinkman RR, Ruttenberg A, He Y: OntoFox: web-based support for ontology reuse. BMC Res Notes 2010, 3:175:1-12.

23. Xiang Z, Zheng J, Lin Y, He Y: Ontorat: automatic generation of new ontology terms, an-notations, and axioms based on ontology design patterns. J Biomed Semantics 2015, 6(1):4 (10 pages).

24. Rubin DL, Noy NF, Musen MA. Protege: a tool for managing and using terminology in radiology applications. J Digit Imaging. 2007;20(Suppl 1):34-46.

25. Ong E, Xiang Z, Zhao B, Liu Y, Lin Y, Zheng J, Mungall C, Courtot M, Ruttenberg A, He Y. Ontobee: a linked ontology data server to support ontology term dereferencing, linkage, query and integration. Nucleic Acids Res. 2017;45(D1):D347-52.

26. Harris S, Seaborne A: SPARQL 1.1 Query Language, W3C Recommendation 21 March 2013. 2013: URL: http://www.w3.org/TR/sparql11-query/, accessed on December 23, 2018.

27. Salvadores M, Alexander PR, Musen MA, Noy NF. BioPortal as a dataset of linked biomedical ontologies and terminologies in RDF. Semantic Web. 2013;4(3):277-84.

28. Smith B, Ceusters W, Klagges B, Kohler J, Kumar A, Lomax J, Mungall C, Neuhaus F, Rector AL, Rosse C. Relations in biomedical ontologies. Genome Biol. 2005:6(5):R46

29. Gibofsky A. Overview of epidemiology, pathophysiology, and diagnosis of rheumatoid arthritis. Am J Manag Care. 2012;18(13 Suppl):S295-302.

30. Van de Wiele T, Van Praet JT, Marzorati M, Drennan MB, Elewaut D. How the microbiota shapes rheumatic diseases. Nat Rev Rheumatol. 2016;12(7):398-411.

31. Stoll ML, Cron RQ. The microbiota in pediatric rheumatic disease: epiphenomenon or therapeutic target? Curr Opin Rheumatol. 2016;28(5):537-43.

32. Caminer AC, Haberman R, Scher JU. Human microbiome, infections, and rheumatic disease. Clin Rheumatol. 2017;36(12):2645-53.

33. Ostrov BE, Amsterdam D. Immunomodulatory interplay of the microbiome and therapy of rheumatic diseases. Immunol Investig. 2017;46(8):769-92.

34. Scher JU, Littman DR, Abramson SB. Microbiome in inflammatory arthritis and human rheumatic diseases. Arthritis Rheumatology. 2016;68(1):35-45.

35. Coit $P$, Sawalha $A H$. The human microbiome in rheumatic autoimmune diseases: a comprehensive review. Clin Immunol. 2016;170:70-9.
36. Rosenbaum JT, Asquith MJ. The microbiome: a revolution in treatment for rheumatic diseases? Curr Rheumatol Rep. 2016;18(10):62.

37. Zhang H, Liao X, Sparks JB, Luo XM. Dynamics of gut microbiota in autoimmune lupus. Appl Environ Microbiol. 2014;80(24):7551-60.

38. Costello ME, Ciccia F, Willner D, Warrington N, Robinson PC, Gardiner B, Marshall M, Kenna TJ, Triolo G, Brown MA. Brief report: intestinal Dysbiosis in Ankylosing spondylitis. Arthritis Rheumatol. 2015;67(3):686-91.

39. Shao T, Shao L, Li H, Xie Z, He Z, Wen C. Combined signature of the fecal microbiome and Metabolome in patients with gout. Front Microbiol. 2017;8:268

40. Stoll ML, Kumar R, Morrow CD, Lefkowitz EJ, Cui X, Genin A, Cron RQ, Elson CO. Altered microbiota associated with abnormal humoral immune responses to commensal organisms in enthesitis-related arthritis. Arthritis Res Ther. 2014;16(6):486

41. Scher JU, Ubeda C, Artacho A, Attur M, Isaac S, Reddy SM, Marmon S, Neimann A, Brusca S, Patel T, et al. Decreased bacterial diversity characterizes the altered gut microbiota in patients with psoriatic arthritis, resembling dysbiosis in inflammatory bowel disease. Arthritis Rheumatol. 2015;67(1):128-39.

42. He Z, Shao T, Li H, Xie Z, Wen C. Alterations of the gut microbiome in Chinese patients with systemic lupus erythematosus. Gut Pathogens. 2016;8:64.

43. Zhang $X$, Zhang D, Jia H, Feng Q, Wang D, Liang D, Wu X, Li J, Tang L, Li Y, et al. The oral and gut microbiomes are perturbed in rheumatoid arthritis and partly normalized after treatment. Nat Med. 2015;21(8):895-905.

44. Field D, Garrity G, Gray T, Morrison N, Selengut J, Sterk P, Tatusova T, Thomson N, Allen MJ, Angiuoli SV, et al. The minimum information about a genome sequence (MIGS) specification. Nat Biotechnol. 2008;26(5):541-7.

45. Yilmaz P, Kottmann R, Field D, Knight R, Cole JR, Amaral-Zettler L, Gilbert JA, Karsch-Mizrachi I, Johnston A, Cochrane G, et al. Minimum information about a marker gene sequence (MIMARKS) and minimum information about any (X) sequence (MIXS) specifications. Nat Biotechnol. 2011;29(5): 415-20

46. Oliveira FS, Brestelli J, Cade S, Zheng J, lodice J, Fischer S, Aurrecoechea C, Kissinger JC, Brunk BP, Stoeckert CJ Jr, et al. MicrobiomeDB: a systems biology platform for integrating, mining and analyzing microbiome experiments. Nucleic Acids Res. 2018;46(D1):D684-91.

47. Duvallet C, Gibbons SM, Gurry T, Irizarry RA, Alm EJ. Meta-analysis of gut microbiome studies identifies disease-specific and shared responses. Nat Commun. 2017:8(1):1784.

48. Crane JK, Naeher TM, Broome JE, Boedeker EC. Role of host xanthine oxidase in infection due to enteropathogenic and Shiga-toxigenic Escherichia coli. Infect Immun. 2013;81(4):1129-39.

49. Crane JK. Role of host xanthine oxidase in infection due to enteropathogenic and Shiga-toxigenic Escherichia coli. Gut Microbes. 2013; 4(5):388-91.

50. Feng Q, Liang S, Jia H, Stadlmayr A, Tang L, Lan Z, Zhang D, Xia H, Xu X, Jie $Z$, et al. Gut microbiome development along the colorectal adenomacarcinoma sequence. Nat Commun. 2015;6:6528.

51. Nougayrede JP, Homburg S, Taieb F, Boury M, Brzuszkiewicz E, Gottschalk G, Buchrieser C, Hacker J, Dobrindt U, Oswald E. Escherichia coli induces DNA double-strand breaks in eukaryotic cells. Science. 2006;313(5788):848-51.

52. Marietta EV, Murray JA, Luckey DH, Jeraldo PR, Lamba A, Patel R, Luthra HS, Mangalam A, Taneja V. Suppression of inflammatory arthritis by human gutderived Prevotella histicola in humanized mice. Arthritis Rheumatol. 2016; 68(12):2878-88

53. The Sixth Annual Workshop of the Clinical and Translational Science Ontology Group, Ann Arbor, MI, USA, October 25-27, 2017 [http://ncorwiki. buffalo.edu/index.php/Microbiology_for_the_CTSA:_Ontological_ Approaches].

54. Delahoy MJ, Omore R, Ayers TL, Schilling KA, Blackstock AJ, Ochieng JB, Moke F, Jaron P, Awuor A, Okonji C, et al. Clinical, environmental, and behavioral characteristics associated with Cryptosporidium infection among children with moderate-to-severe diarrhea in rural western Kenya, 20082012: the global enteric multicenter study (GEMS). PLoS Negl Trop Dis. 2018; 12(7):e0006640.

55. Kotloff KL, Blackwelder WC, Nasrin D, Nataro JP, Farag TH, van Eijk A, Adegbola RA, Alonso PL, Breiman RF, Faruque AS, et al. The Global Enteric Multicenter Study (GEMS) of diarrheal disease in infants and young children in developing countries: epidemiologic and clinical methods of the case/ control study. Clin Infect Dis. 2012;55(Suppl 4):S232-45. 
56. Huang da W, Sherman BT, Lempicki RA. Bioinformatics enrichment tools: paths toward the comprehensive functional analysis of large gene lists. Nucleic Acids Res. 2009;37(1):1-13.

57. Badal VD, Wright D, Katsis Y, Kim HC, Swafford AD, Knight R, Hsu CN.

Challenges in the construction of knowledge bases for human microbiomedisease associations. Microbiome. 2019;7(1):129.

\section{Publisher's Note}

Springer Nature remains neutral with regard to jurisdictional claims in published maps and institutional affiliations.

Ready to submit your research? Choose BMC and benefit from:

- fast, convenient online submission

- thorough peer review by experienced researchers in your field

- rapid publication on acceptance

- support for research data, including large and complex data types

- gold Open Access which fosters wider collaboration and increased citations

- maximum visibility for your research: over $100 \mathrm{M}$ website views per year

At $\mathrm{BMC}$, research is always in progress.

Learn more biomedcentral.com/submissions 